\title{
INVARIANTS AND COINVARIANTS OF FINITE PSEUDOREFLECTION GROUPS, JACOBIAN DETERMINANTS AND STEENROD OPERATIONS
}

\author{
LARRY SMITH \\ AG-Invariantentheorie Math Institut, Bunsenstraße 3-5 D 37073 Göttingen, Germany \\ and Mathematical Sciences Department, University of Aberdeen \\ (larry@sunrise.uni-math.gwdg.de)
}

(Received 11 January 2000)

\begin{abstract}
Let $\rho: G \hookrightarrow \operatorname{GL}(n, \mathbb{F})$ be a representation of a finite group $G$ over a finite field $\mathbb{F}$ and $f_{1}, \ldots, f_{n} \in \mathbb{F}[V]^{G}$ such that the ring of invariants is a polynomial algebra $\mathbb{F}\left[f_{1}, \ldots, f_{n}\right]$. It is known that in this case the algebra of coinvariants $\mathbb{F}[V]_{G}$ is a Poincaré duality algebra, and if, moreover, the order of $G$ is invertible in $\mathbb{F}$, that a fundamental class is represented by the Jacobian determinant $\operatorname{det}\left[\partial f_{i} / \partial z_{j}\right]$, and is therefore a $\operatorname{det}^{-1}$-relative invariant. In this note we deduce what happens in the modular case. As a bonus we obtain a new criterion for an unstable algebra over the Steenrod algebra to be a ring of invariants.
\end{abstract}

Keywords: rings of coinvariants; reflection groups; Steenrod operations; Jacobian determinants; Poincaré duality algebras; relative invariants

AMS 2000 Mathematics subject classification: Primary 13A50; 55S10

\section{Introduction}

Let $\rho: G \hookrightarrow \mathrm{GL}(n, \mathbb{F})$ be a representation of a finite group over the field $\mathbb{F}$, and let $V=\mathbb{F}^{n}$ be the corresponding $G$-module. Via $\rho$ the group $G$ acts on the symmetric algebra $S\left(V^{*}\right)=\mathbb{F}[V]$ of the dual representation $V^{*}$, which may be regarded as the algebra of polynomial functions on $V$. The subalgebra $\mathbb{F}[V]^{G} \subseteq \mathbb{F}[V]$ of polynomial functions fixed under the $G$-action is called the ring of invariants of $G$, or perhaps better $\rho$. As a general reference for invariant theory we use [15], the notation and conventions of which we adhere to. The Steenrod algebra, $\mathcal{P}^{*}$, of a Galois field and its basic properties [15, Chapters 10 and 11] are used in $\S 2$, often without explicit reference, and a certain familiarity with the Steenrod algebra is therefore assumed.

The study of the representations $\rho$ for which $\mathbb{F}[V]^{G} \subseteq \mathbb{F}[V]$ is itself a polynomial algebra has played a central role in invariant theory. In this case it is known that the ring of coinvariants, $\mathbb{F}[V]_{G}=\mathbb{F} \otimes_{\mathbb{F}[V] G} \mathbb{F}[V]$, is a Poincaré duality algebra (see, for example, $[\mathbf{1 4}]$ or $[\mathbf{1 5}$, Theorem 6.5.1]). Moreover, in the non-modular case the Jacobian determinant $\operatorname{det}\left[\partial f_{i} / \partial z_{j}\right]$ is a representative for a fundamental class, which is therefore a $\operatorname{det}^{-1}$-relative invariant $[\mathbf{1 5}]$. In the modular case the Jacobian determinant may fail 
to represent a fundamental class: this happens for example for the representation of $\mathbb{Z} / p$ implemented by the matrix

$$
\left[\begin{array}{ll}
1 & 1 \\
0 & 1
\end{array}\right] \in \mathrm{GL}\left(2, \mathbb{F}_{p}\right) .
$$

If $x, y$ is the standard dual basis, then $\mathbb{F}_{p}[x, y]^{\mathbb{Z} / p}=\mathbb{F}_{p}\left[x\left(x^{p-1}-y^{p-1}\right), y\right]$ and the Jacobian determinant of the generators is $y^{p-1}$, which is zero in the algebra of coinvariants $\mathbb{F}_{p}[x, y]_{G} \cong \mathbb{F}_{p}[x, y] /\left(x^{p}, y\right) \cong \mathbb{F}[x] /\left(x^{p}\right)$ (see, for example, [15, Chapter 8, $\left.\S 3\right]$, the example of the unipotent subgroup of $\left.\operatorname{GL}\left(n, \mathbb{F}_{p}\right)\right)$. This raises several questions, or problems, for which it would be interesting to have characteristic free solutions. Namely, suppose that $\rho: G \hookrightarrow \operatorname{GL}(n, \mathbb{F})$ is a representation of a finite group for which $\mathbb{F}[V]^{G}=\mathbb{F}\left[f_{1}, \ldots, f_{n}\right]$ is a polynomial algebra.

Problem 1.1. Is the Jacobian determinant $\operatorname{det}\left[\partial f_{i} / \partial z_{j}\right]$ at least non-zero in $\mathbb{F}[V]$ ?

As noted above, the Jacobian determinant, when regarded as an element of $\mathbb{F}[V]$, is a $\operatorname{det}^{-1}$-relative invariant, i.e.

$$
g\left(\operatorname{det}\left[\frac{\partial f_{i}}{\partial z_{j}}\right]\right)=\operatorname{det}(g)^{-1} \cdot \operatorname{det}\left[\frac{\partial f_{i}}{\partial z_{j}}\right]
$$

so, if it represents a fundamental class of $\mathbb{F}[V]_{G}$, then such a class is likewise a $\operatorname{det}^{-1}$ relative invariant.

Problem 1.2. Is a fundamental class $\left[\mathbb{F}[V]_{G}\right]$ of $\mathbb{F}[V]_{G}$ always a $\operatorname{det}^{-1}$-relative invariant?

As it turns out, the answer to both these questions is in the affirmative: for Problem 1.1 the result would seem to be due to Benson [2, Proposition 5.4.2], and the proof is a delicate piece of field theory. We will prove as part of Theorem 2.1 that the corresponding statement for the Milnor primitives in the Steenrod algebra holds. This is considerably more elementary, and implies the affirmative solution to Problem 1.1 for Galois fields. It also leads to a new criterion for an unstable polynomial algebra over the Steenrod algebra to be a ring of invariants (Theorem 2.2). The solution (Theorem 3.1) to Problem 1.2 is also not difficult, though a bit delicate, and is based on the fact [4, Chapter V, $\S 6$, Exercise 8] that groups whose rings of invariants are polynomial algebras are generated by pseudoreflections, and the author's description [14] of a fundamental class for $\mathbb{F}[V]_{G}$. This leaves the last problem, as follows.

Problem 1.3. When is $\operatorname{det}\left[\partial f_{i} / \partial z_{j}\right]$ a representative for a fundamental class of $\mathbb{F}[V]_{G}$ ?

The fickleness of Jacobians is that the answer to the last question depends on the characteristic of $\mathbb{F}$, the order $|G|$ of $G$, and the representation $\rho$. This is the content of Theorem 4.6. 


\section{Jacobian determinants}

Let $\rho: G \hookrightarrow \operatorname{GL}(n, \mathbb{F})$ be a representation of a finite group over the field $\mathbb{F}$, and let $V=\mathbb{F}^{n}$ be the corresponding $G$-module. There are some well-known criteria, such as [15, Theorems 5.5.5 and 7.3.2], for the ring of invariants $\mathbb{F}[V]^{G}$ to be a polynomial algebra. Less well known is the result of Kemper [8, Proposition 16] using Jacobians, one direction of which is a rather intricate field theoretic result [2, Proposition 5.4.2]. Kemper's result [8] is not directly applicable to an abstract polynomial algebra over the Steenrod algebra, $\mathcal{P}^{*}$, as it requires an integral embedding of such an algebra in a polynomial algebra on linear generators to even speak of the Jacobian determinant (see $[\mathbf{1 2}]$ for more details).

In this section we will restrict our attention to the case where $\mathbb{F}$ is a Galois field, i.e. a finite field with $q=p^{\nu}$ elements, where $p \in \mathbb{N}$ is a prime integer, and prove the analogue of Benson's field theoretic result [2] for Steenrod operations. By an elementary changeof-basis argument we also recover the field theoretic result, at least for finite fields. The determinant criteria with the Milnor primitives in the Steenrod algebra can be used to provide an embedding-free characterization of when an unstable polynomial algebra over the Steenrod algebra $\mathcal{P}^{*}$ is a ring of invariants.

As it turns out it is convenient to prove both determinant results at once.

Notation. Throughout this section $\mathbb{F}=\mathbb{F}_{q}$ denotes the Galois field with $q=p^{\nu}$ elements, where $p \in \mathbb{N}$ is a prime integer.

Theorem 2.1. Let $\rho: G \hookrightarrow \operatorname{GL}(n, \mathbb{F})$ be a representation of a finite group $G$ over the finite field $\mathbb{F}$, and let $\mathbb{F}[V]^{G}$ be the algebra of $G$-invariant polynomial functions on $V$. Suppose that $\mathbb{F}[V]^{G}=\mathbb{F}\left[f_{1}, \ldots, f_{n}\right]$ is a polynomial algebra. If $P^{\Delta_{i}} \in \mathcal{P}^{*}$ is the Milnor primitive element of degree $q^{i}-1$, then

$$
\operatorname{det}\left[P^{\Delta_{i}}\left(f_{j}\right)\right] \neq 0 \in \mathbb{F}[V] .
$$

If $z_{1}, \ldots, z_{n} \in V^{*}$ is a basis for the space of linear forms, then the Jacobian determinant $\operatorname{det}\left[\partial f_{i} / \partial z_{j}\right]$ is non-zero in $\mathbb{F}[V]$ also.

Proof. We begin by considering the universal example, namely $G=\mathrm{GL}(n, \mathbb{F})$ for which the ring of invariants is the Dickson algebra $\mathbb{F}[V]^{\mathrm{GL}(n, \mathbb{F})}=\mathrm{D}_{*}(n)$. Recall that $\mathrm{D}_{*}(n)=\mathbb{F}\left[\mathrm{d}_{n, 0}, \ldots, \mathrm{d}_{n, n-1}\right]$, where $\mathrm{d}_{n, 0}, \ldots, \mathrm{d}_{n, n-1}$ are the Dickson polynomials. From [17] (see also the proof of Theorem 10.6.8 in $[\mathbf{1 5}]$, where the $y_{k}$ correspond to $\mathrm{d}_{n, n-k}$ ) we have

$$
\operatorname{det}\left[P^{\Delta_{i}}\left(\mathrm{~d}_{n, n-k}\right)\right]=\mathrm{d}_{n, 0}^{n} \neq 0
$$

and so it remains to show that

$$
\operatorname{det}\left[\frac{\partial \mathrm{d}_{n, i}}{\partial z_{j}}\right]_{\substack{i=0, \ldots, n-1 \\ j=1, \ldots, n}} \neq 0 .
$$

to complete the proof for this case. 
To this end we need the formula (see [6] for details) between operators*

$$
P^{\Delta_{i}}=\sum_{j=1}^{n} z_{j}^{q^{i}} \frac{\partial}{\partial z_{j}}, \quad i=1,2, \ldots
$$

This formula is verified by observing that

(1) it holds for linear forms,

(2) both sides are derivations, so satisfy the Leibniz rule,

(3) every polynomial is a sum of monomials, and

(4) every monomial is a product of linear forms.

In matrix form this says

$$
\left[\begin{array}{c}
P^{\Delta_{1}} \\
\vdots \\
P^{\Delta_{n}}
\end{array}\right]=\left[z_{j}^{q^{i}}\right] \cdot\left[\begin{array}{c}
\frac{\partial}{\partial z_{1}} \\
\vdots \\
\frac{\partial}{\partial z_{n}}
\end{array}\right] .
$$

The matrix $\left[z_{i}^{q^{j}}\right]$ has non-zero determinant, e.g. because the coefficient of the monomial $z_{1}^{q} z_{2}^{q^{2}} \ldots z_{n}^{q^{n}}$ in the expansion is 1 . Therefore, over the field of fractions $\mathbb{F}(V)$ of $\mathbb{F}[V]$, the preceding linear system can be solved (e.g. by Cramer's rule) to express $\partial / \partial z_{j}$ as a linear combination of $P^{\Delta_{1}}, \ldots, P^{\Delta_{n}}$, say

$$
\frac{\partial}{\partial z_{j}}=\sum_{i=1}^{n} w_{j, i} P^{\Delta_{i}}, \quad w_{j, i} \in \mathbb{F}(V) .
$$

Applying this to the Dickson polynomials $\mathrm{d}_{n, 0}, \ldots, \mathrm{d}_{n, n-1}$ we obtain for the Jacobian determinant the formula

$$
\operatorname{det}\left[\frac{\partial \mathrm{d}_{n, k}}{\partial z_{j}}\right]=\operatorname{det}\left[w_{j, i}\right] \cdot \operatorname{det}\left[P^{\Delta_{i}}\left(\mathrm{~d}_{n, k}\right)\right] \neq 0
$$

Since both

$$
\operatorname{det}\left[w_{j, i}\right]=\operatorname{det}\left[z_{i}^{q^{j}}\right]^{-1} \text { and } \operatorname{det}\left[P^{\Delta_{i}}\left(\mathrm{~d}_{n, k}\right)\right]=\mathrm{d}_{n, 0}^{n},
$$

are non-zero, we conclude that $\operatorname{det}\left[\partial f_{i} / \partial z_{j}\right] \neq 0$ also, as required.

The general case follows from the chain rule: we have the inclusion $\mathrm{D}_{*}(n) \subseteq \mathbb{F}[V]^{G}=$ $\mathbb{F}\left[f_{1}, \ldots, f_{n}\right]$, and hence each Dickson polynomial may be regarded as a polynomial in $f_{1}, \ldots, f_{n}$. Doing so we may apply the chain rule for Jacobian matrices to obtain

$$
\left[\frac{\partial \mathrm{d}_{n, k}}{\partial z_{j}}\right]=\left[\frac{\partial \mathrm{d}_{n, k}}{\partial f_{i}}\right] \cdot\left[\frac{\partial f_{i}}{\partial z_{j}}\right]
$$

* In other words this formula holds in $\operatorname{End}(\mathbb{F}[\cdot])$, the algebra of linear endomorphisms of the functor $\operatorname{Vect}_{\mathbb{F}} \rightsquigarrow \operatorname{Vect}_{\mathbb{F}}$ given by $V \rightsquigarrow \mathbb{F}[V]$. 
Taking determinants then gives

$$
\operatorname{det}\left[\frac{\partial \mathrm{d}_{n, k}}{\partial z_{j}}\right]=\operatorname{det}\left[\frac{\partial \mathrm{d}_{n, k}}{\partial f_{i}}\right] \cdot \operatorname{det}\left[\frac{\partial f_{i}}{\partial z_{j}}\right],
$$

from which we conclude that

$$
\operatorname{det}\left[\frac{\partial f_{i}}{\partial z_{j}}\right] \neq 0
$$

Finally, the formula

$$
\operatorname{det}\left[P^{\Delta_{i}}\left(f_{j}\right)\right]=\operatorname{det}\left[z_{j}^{q^{i}}\right] \cdot \operatorname{det}\left[\frac{\partial f_{i}}{\partial z_{j}}\right]
$$

shows that $\operatorname{det}\left[P^{\Delta_{i}}\left(f_{j}\right)\right] \neq 0$ as desired.

This provides us with a new proof of the affirmative answer to Problem 1.1 for finite fields. Notice that, in contrast to Kemper's condition [8], the non-vanishing of $\operatorname{det}\left(P^{\Delta_{i}}\left(f_{j}\right)\right)$ does not depend on an embedding in $\mathbb{F}[V]$. This result can therefore be used to provide a succinct, necessary and sufficient condition: that an unstable polynomial algebra over the Steenrod algebra be a ring of invariants. Namely the following theorem.

Theorem 2.2. Let $H=\mathbb{F}\left[u_{1}, \ldots, u_{n}\right]$ be an unstable polynomial algebra over the Steenrod algebra $\mathcal{P}^{*}$ of $\mathbb{F}$. Then $H$ is the ring of invariants of a finite pseudoreflection group $G \leqslant \mathrm{GL}(n, \mathbb{F})$ if and only if

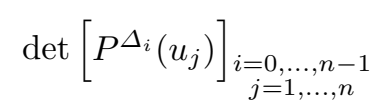

is non-zero.

Proof. Suppose that $u_{1}, \ldots, u_{n}$ satisfy

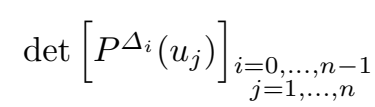

is non-zero. According to the Galois Embedding Theorem of [12] it suffices to show that $H$ is $\mathcal{P}^{*}$-inseparably closed to conclude that it is a ring of invariants. So suppose $h \in H$ and $P^{\Delta_{k}}(h)=0$ for $k=0,1, \ldots$ We need to show that $h$ is a $p$ th power in $H$.

Since $\mathbb{F}$ is a finite field, it is perfect, and hence an element $h$ in $H$ is a $p$ th power if and only if $\partial h / \partial u_{i}=0$ for $i=1, \ldots, n$. Recall that each of the sets of $n$ derivations $[\mathbf{1 2}, \S 1.1]$,

$$
\begin{aligned}
& \frac{\partial}{\partial u_{i}}, \quad i=1, \ldots, n, \\
& P^{\Delta_{k}}, \quad k=0, \ldots, n-1,
\end{aligned}
$$


are a basis over $F F(H)$, the field of fractions of $H$, for the module of derivations of $H$ with values in its field of fractions $F F(H)$. Therefore we can express $\partial / \partial u_{j}$ as a linear combination

$$
\frac{\partial}{\partial u_{j}}=\sum_{k=0}^{n-1} U_{i, k} P^{\Delta_{k}}
$$

of $P^{\Delta_{k}}$ for $k=0,1, \ldots, n-1$ with coefficients $U_{i, k} \in F F(H)$. Then $P^{\Delta_{k}}(h)=0$ for $k=0,1, \ldots, n-1$ entails that $\partial h / \partial u_{i}=0$ for $i=1, \ldots, n$, and hence $h$ is a $p$ th power, as was to be shown.

The converse follows from the previous proposition.

The condition that

$$
\operatorname{det}\left[P^{\Delta_{i}}\left(f_{j}\right)\right] \neq 0
$$

for an unstable polynomial algebra $H=\mathbb{F}\left[f_{1}, \ldots, f_{n}\right]$ is non-vacuous, as the following somewhat artificial examples show. For, if $\rho: G \hookrightarrow \operatorname{GL}(n, \mathbb{F})$ has $\mathbb{F}_{q}\left[f_{1}, \ldots, f_{n}\right]$ as a ring of invariants, then the ring of invariants of $G$ acting on $\mathbb{F}_{q}\left[z_{1}^{q}, \ldots, z_{n}^{q}\right]$ will be $\mathbb{F}_{q}\left[f_{1}^{\xi}, \ldots, f_{n}^{\xi}\right]$, where

$$
f_{i}^{\xi}\left(z_{1}, \ldots, z_{n}\right)=f\left(z_{1}^{q}, \ldots, z_{n}^{q}\right) \in \mathbb{F}_{q}\left[z_{1}, \ldots, z_{n}\right]
$$

for $i=1, \ldots, n$. Alone for degree reasons $P^{\Delta_{0}}$ and $P^{\Delta_{1}}$ vanish on $\mathbb{F}_{q}\left[f_{1}^{\xi}, \ldots, f_{n}^{\xi}\right]$, so $\operatorname{det}\left[P^{\Delta_{i}}\left(f_{j}^{\xi}\right)\right]=0$. If $q=2$, then examples of this type occur naturally in algebraic topology, for example, as $H^{*}\left(\boldsymbol{B} \mathbb{S P}(n) ; \mathbb{F}_{2}\right)=\mathbb{F}_{2}\left[\mathfrak{p}_{1}^{\mathbb{S P}}, \ldots, \mathfrak{p}_{n}^{\mathbb{S P}}\right]$, where $\mathfrak{p}_{i}^{\mathbb{S P}}$ is the $i$ th elementary symmetric function in the fourth power of $n$ linear variables. This raises a number of points, e.g. to what extent are unstable polynomial algebras $H=\mathbb{F}_{q}\left[f_{1}, \ldots, f_{n}\right]$, where $\operatorname{det}\left[P^{\Delta_{i}}\left(f_{j}\right)\right]=0$, of this form? (See [20] for more details.) To close this discussion, we note that as a consequence of Theorem 2.2 and Proposition 7.1.1 in [12] we have the following corollary.

Corollary 2.3. If $H=\mathbb{F}_{q}\left[f_{1}, \ldots, f_{n}\right]$ is an unstable polynomial algebra over $\mathcal{P}^{*}$ and $P^{\Delta_{i}}$ is identically zero for some $i \in \mathbb{N}_{0}$, then $H$ cannot be realized as a ring of invariants.

\section{Fundamental classes}

We turn next to the second problem posed in $\S 1$. In this section $\mathbb{F}$ will be an arbitrary field, $\rho: G \hookrightarrow \mathrm{GL}(n, \mathbb{F})$ will be a representation of a finite group $G$ over $\mathbb{F}$, and $\mathbb{F}[V]_{G}$ will be the ring of coinvariants. (For basic facts about rings of coinvariants, see, for example, $[\mathbf{1 5}, \S 1.3]$.) It is known that when $\mathbb{F}[V]^{G}=\mathbb{F}\left[f_{1}, \ldots, f_{n}\right]$ is a polynomial algebra then $\mathbb{F}[V]_{G}$ is a Poincaré duality algebra (see $[\mathbf{1 4}]$ and $[\mathbf{1 5}$, Theorem 6.5.1]). In the nonmodular case, i.e. when ${ }^{*}|G| \in \mathbb{F}^{\times}$, this is a well-studied finite-dimensional representation of $G$. In particular, one knows it is isomorphic to the regular representation of $G$ over $\mathbb{F}$ (see, for example, [5] or [15, Theorem 7.5.2]) and a fundamental class is represented by $\operatorname{det}\left[\partial f_{i} / \partial z_{j}\right]$, and so is a $\operatorname{det}^{-1}$-relative invariant. The purpose of this section is to prove that the last of these conclusions, namely that the fundamental class of $\mathbb{F}[V]_{G}$ is a

\footnotetext{
* $|G|$ denotes the order of $G$.
} 
$\operatorname{det}^{-1}$-relative invariant, holds in general. The proof is characteristic free, and provides a new proof of this fact even in the non-modular case.

Theorem 3.1. Let $\rho: G \hookrightarrow \operatorname{GL}(n, \mathbb{F})$ be a representation of a finite group $G$ over the field $\mathbb{F}$. If $\mathbb{F}[V]^{G}$ is a polynomial algebra, then the fundamental class, $\left[\mathbb{F}[V]_{G}\right]$, of the ring of coinvariants is a $\operatorname{det}^{-1}$-relative invariant.

Proof. Choose homogeneous polynomials $f_{1}, \ldots, f_{n} \in \mathbb{F}[V]$ such that

$$
\mathbb{F}[V]^{G} \cong \mathbb{F}\left[f_{1}, \ldots, f_{n}\right] .
$$

We begin by recalling from $[\mathbf{1 4}]$ how to compute a fundamental class for $\mathbb{F}[V]_{G}$, or, indeed, for $\mathbb{F}\left[z_{1}, \ldots, z_{n}\right] /\left(f_{1}, \ldots, f_{n}\right)$, whenever $f_{1}, \ldots, f_{n} \in \mathbb{F}\left[z_{1}, \ldots, z_{n}\right]$ is a system of parameters, and $z_{1}, \ldots, z_{n}$ is a basis for $V^{*}$, the space of linear forms. Here is how this goes: write

$$
f_{i}=\sum_{j=1}^{n} a_{i, j} z_{j}, \quad i=1, \ldots, n
$$

for homogeneous polynomials $a_{i, j} \in \mathbb{F}\left[z_{1}, \ldots, z_{n}\right], i, j=1, \ldots, n$. The determinant, $\operatorname{det}\left[a_{i, j}\right]$, regarded as an element of the quotient algebra $\mathbb{F}\left[z_{1}, \ldots, z_{n}\right] /\left(f_{1}, \ldots, f_{n}\right)$, is a fundamental class.

To begin the proof proper, we recall $[4$, Chapter $\mathrm{V}, \S 6$, Exercise 8$]$ that, since $\mathbb{F}[V]^{G}$ is a polynomial algebra, $G$ (or better, put $\rho(G)$ ) is generated by pseudoreflections. Therefore, to show that the fundamental class of $\mathbb{F}[V]_{G}$ is a $\operatorname{det}^{-1}$-relative invariant, it will suffice to show that

$$
s\left(\left[\mathbb{F}[V]_{G}\right]\right)=\operatorname{det}(s)^{-1} \cdot\left[\mathbb{F}[V]_{G}\right] \in \mathbb{F}[V]_{G}
$$

for all pseudoreflections $s \in G$. The pseudoreflections in $G$ can be of two types: diagonalizable or transvections. We consider these two cases separately.

Case 1. $s \in s_{\Delta}(G)$, i.e. $s$ is diagonalizable.

We choose a basis $z_{1}, \ldots, z_{n}$ for $V^{*}$ so that $s$ is represented by the matrix

$$
\left[\begin{array}{ccccc}
1 & 0 & \ldots & \ldots & 0 \\
0 & 1 & 0 & \ldots & 0 \\
0 & & \ddots & \ldots & 0 \\
0 & \ldots & \ldots & 1 & 0 \\
\vdots & \ldots & \ldots & 0 & \lambda
\end{array}\right] \in \operatorname{GL}(n, \mathbb{F}), \quad \lambda \in \mathbb{F}^{\times} .
$$

Then

$$
s\left(z_{i}\right)= \begin{cases}z_{i} & \text { for } i=1, \ldots, n-1, \\ \lambda z_{n} & \text { for } i=n .\end{cases}
$$

Choose $a_{i, j} \in \mathbb{F}[V]$ such that

$$
f_{i}=\sum_{i=1}^{n} a_{i, j} z_{j}, \quad i=1, \ldots, n .
$$


This way of writing $f_{i}$ is not unique, and we choose to write it in this way so that none of the polynomials $a_{i, j}$ for $j \neq n$ is divisible by $z_{n}$. This is possible because, if

$$
a_{i, j}=\sum_{E=\left(e_{1}, \ldots, e_{n-1}, 0\right)} \alpha_{E} z^{E}+\sum_{K=\left(k_{1}, \ldots, k_{n}\right) \mid k_{n} \neq 0} \beta_{K} z^{K},
$$

then the desired form can be achieved by using the equalities

$$
\beta_{K} z^{K} z_{j}=\beta_{K} z^{K+\Delta_{j}-\Delta_{n}} z_{n}, \quad j=1, \ldots, n-1,
$$

where $\Delta_{k}$ is the exponent sequence with a 1 in the $k$ th position and zeros elsewhere.

Having done this, we note that it means

$$
a_{i, j} \in \mathbb{F}\left[z_{1}, \ldots, z_{n-1}\right], \quad \text { for } j=1, \ldots, n-1, \quad \text { and } i=1, \ldots, n,
$$

and hence from (3.1) we get

$$
s\left(a_{i, j}\right)=a_{i, j}, \quad \text { for } j=1, \ldots, n-1, \quad \text { and } \quad i=1, \ldots, n .
$$

If we apply $s$ to (3.2) and use (3.3) we get

$$
\begin{aligned}
f_{i} & =s f_{i}=\sum_{j=1}^{n} s\left(a_{i, j}\right) s\left(z_{j}\right) \\
& =\sum_{j=1}^{n-1} a_{i, j} z_{j}+\lambda s\left(a_{i, n}\right) z_{n},
\end{aligned}
$$

since $f_{i}$ is invariant for $i=1, \ldots, n$. Equating this representation for $f_{i}$ with (3.2) and simplifying gives

$$
a_{i, n} z_{n}=\lambda s\left(a_{i, n}\right) z_{n}, \quad \text { for } i=1, \ldots, n,
$$

from which we conclude

$$
s\left(a_{i, n}\right)=\lambda^{-1} a_{i, n}, \quad \text { for } i=1, \ldots, n .
$$

Therefore

$$
\operatorname{det}\left[s\left(a_{i, j}\right)\right]=\operatorname{det}\left[\begin{array}{cccc}
a_{1,1} & \ldots & a_{1, n-1} & \lambda^{-1} a_{1, n} \\
\vdots & \vdots & \vdots & \vdots \\
a_{n, 1} & \ldots & a_{n, n-1} & \lambda^{-1} a_{n, n}
\end{array}\right]=\lambda^{-1} \operatorname{det}\left[a_{i, j}\right]
$$

by an elementary property of determinants $[\mathbf{1 3}$, p. 240]. Since $\operatorname{det}(s)=\lambda$, and we may choose $\left[\mathbb{F}[V]_{G}\right]=\operatorname{det}\left[a_{i, j}\right]$, we have shown

$$
s\left(\left[\mathbb{F}[V]_{G}\right]\right)=\operatorname{det}(s)^{-1}\left[\mathbb{F}[V]_{G}\right],
$$

as was claimed. 
Case 2. $t \in s_{\not \triangle}(G)$, i.e. $t$ is a transvection.

We choose a basis $z_{1}, \ldots, z_{n}$ for $V^{*}$ such that the matrix of $t$ is

$$
\left[\begin{array}{cccc}
1 & 0 & \ldots & 0 \\
0 & \ddots & & 0 \\
0 & \vdots & 1 & 1 \\
0 & \ldots & \ldots & 1
\end{array}\right] \in \operatorname{GL}(n, \mathbb{F})
$$

in other words,

$$
t\left(z_{i}\right)= \begin{cases}z_{i} & \text { for } i=1, \ldots, n-1, \\ z_{n}+z_{n-1} & \text { for } i=n .\end{cases}
$$

This time we choose to write

$$
f_{i}=\sum_{j=1}^{n} a_{i, j} z_{j}, \quad \text { for } i=1, \ldots, n
$$

in such a way that

$$
a_{i, j} \in \mathbb{F}\left[z_{1}, \ldots, z_{n-2}, z_{n}\right], \quad \text { for } j=1, \ldots, n-2, n \quad \text { and } \quad i=1, \ldots, n \text {. }
$$

As noted in the previous case this sort of rewriting is always possible. If we apply $t$ to (3.5), use (3.4), and that $f_{i}$ is invariant for $i=1, \ldots, n$, we obtain

$$
\begin{aligned}
f_{i} & =t\left(f_{i}\right)=\sum_{j=1}^{n} t\left(a_{i, j}\right) t\left(z_{j}\right) \\
& =\sum_{j=1}^{n-2} a_{i, j} z_{j}+t\left(a_{i, n-1}\right) z_{n-1}+a_{i, n}\left(z_{n}+z_{n-1}\right) \\
& =\sum_{j=1}^{n-2} a_{i, j} z_{j}+\left(t\left(a_{i, n-1}\right)+a_{i, n}\right) z_{n-1}+a_{i, n} z_{n} .
\end{aligned}
$$

Equating with (3.5) gives, with the use of (3.6) after simplification,

$$
a_{i, n-1} z_{n-1}=\left(t\left(a_{i, n-1}\right)+a_{i, n}\right) z_{n-1},
$$

from which we conclude

$$
t\left(a_{i, n-1}\right)=a_{i, n-1}-a_{i, n}, \quad \text { for } i=1, \ldots, n .
$$

Hence, from (3.5) and (3.7) we obtain

$$
\operatorname{det}\left[t\left(a_{i, j}\right)\right]=\operatorname{det}\left[\begin{array}{cccc}
a_{1,1} & \ldots & a_{1, n-1}-a_{1, n} & a_{1, n} \\
\vdots & \vdots & \vdots & \vdots \\
a_{n, 1} & \ldots & a_{n, n-1}-a_{n, n} & a_{n, n}
\end{array}\right]=\operatorname{det}\left[a_{i, j}\right]
$$


by the same elementary property of determinants as in the previous case [13, p. 240]. Since we may choose to represent $\left[\mathbb{F}[V]_{G}\right]$ by $\operatorname{det}\left[a_{i, j}\right]$ and $\operatorname{det}(t)=1$ we have shown that

$$
t\left(\left[\mathbb{F}[V]_{G}\right]\right)=\operatorname{det}(t)^{-1}\left[\mathbb{F}[V]_{G}\right],
$$

as was claimed.

Since $G$ is generated by pseudoreflections it follows that $g\left(\left[\mathbb{F}[V]_{G}\right]\right)=\operatorname{det}(g)^{-1}\left[\mathbb{F}[V]_{G}\right]$ for any $g \in G$.

\section{Jacobians and fundamental classes}

This section is devoted to the last problem posed in $\S 1$, where the answer is decidedly characteristic and representation dependent. To begin, we let $\mathbb{F}$ denote an arbitrary field, introduce some notations, and review some material (from $[\mathbf{1 1}]$ and $[\mathbf{1 5}, \S 7.1]$ ), before turning to the task at hand.

Let $s \in \mathrm{GL}(n, \mathbb{F})$ be a pseudoreflection. The reflecting hyperplane $V^{s} \subsetneq V$ of $s$ will be denoted by $H_{s}$. The set of linear forms in $V^{*}$ with kernel $H_{s}$ is one dimensional, and $\ell_{s}$ will denote an arbitrary, but fixed, linear form with $\operatorname{ker}\left(\ell_{s}\right)=H_{s}$. Since $s$ fixes a hyperplane pointwise, 1 is an eigenvalue of $s$ of multiplicity at least $n-1$, so the characteristic polynomial of $s$ splits into linear factors. The remaining eigenvalue, which could also be 1 , is denoted by $\lambda_{s}$. Note that $\operatorname{det}(s)=\lambda_{s}$. Associated with $s$ is a twisted differential operator $\Delta_{s}$, introduced by Chevalley in [5], with the properties

$$
\left.\begin{array}{rl}
\Delta_{s}(f) & =(s f-f) \cdot \ell_{s}, \\
\Delta_{s}(f h) & =\Delta_{s}(f) h+s(f) \Delta_{s}(h),
\end{array}\right\}
$$

for any $f, h \in \mathbb{F}[V]$ (see, for example, $[\mathbf{1 1}]$ or $[\mathbf{1 5}, \S 7.1]$ ). With this notation one has

$$
\begin{aligned}
\Delta_{s}\left(\ell_{s}\right) & =\lambda_{s}-1, \\
s\left(\ell_{s}\right) & =\lambda_{s} \cdot \ell_{s} .
\end{aligned}
$$

From this one obtains

$$
\Delta_{s}\left(\ell_{s}^{k}\right)=\left(1+\lambda_{s}+\cdots+\lambda_{s}^{k-1}\right)\left(\lambda_{s}-1\right) \ell_{s}^{k-1}=\left(\lambda_{s}^{k}-1\right) \ell_{s}^{k-1},
$$

by induction, and therefore it follows that

$$
\Delta_{s}^{k}\left(\ell_{s}^{k}\right)=\left(\lambda_{s}^{k}-1\right) ! \in \mathbb{F} .
$$

In particular, if $\lambda_{s} \in \mathbb{F}^{\times}$has order $\left|\lambda_{s}\right|$, then

$$
\Delta_{s}^{\left|\lambda_{s}\right|-1}\left(\ell_{s}^{\left|\lambda_{s}\right|-1}\right)=\left(\lambda_{s}^{\left|\lambda_{s}\right|-1}-1\right) ! \neq 0 \in \mathbb{F} .
$$

If $\rho: G \hookrightarrow \mathrm{GL}(n, \mathbb{F})$ is a representation of a finite group $G$ over the field $\mathbb{F}$, we denote by $s(G) \subset G$ the set of pseudoreflections in $G$, i.e. the set of $s \in G$ such that $\rho(s)$ is a pseudoreflection. The diagonalizable pseudoreflections in $s(G)$, i.e. those $s \in s(G)$ with 
$\lambda_{s} \neq 1$, are denoted by $s_{\Delta}(G)$, and the non-diagonalizable pseudoreflections, also called transvections, by $s_{\Delta}(G)$. The transvections together with $1 \in G$ form a normal subgroup of $s(G)$, the kernel of the homomorphism det $: s(G) \rightarrow \mathbb{F}^{\times}$.

Likewise, $\mathcal{H}(G)=\left\{H_{s} \mid s \in s(G)\right\}$ denotes the set of reflecting hyperplanes of the pseudoreflections in $G$, with $\mathcal{H}_{\Delta}(G)$ the subset of $\mathcal{H}(G)$ where $s$ is in $s_{\Delta}(G)$, and $\mathcal{H}_{\Delta}(G)$ the subset of $\mathcal{H}(G)$ where $s$ is in $s_{\Delta}(G)$. If $H \in \mathcal{H}(G)$, we denote by $\ell_{H}$ a linear form with kernel $H$. If $\mathbb{F}$ is a field of characteristic $p \neq 0$, then the set of all pseudoreflections in $G$ with reflecting hyperplane $H$ is a semidirect product of an elementary abelian $p$-group, the subgroup of transvections, and a cyclic group of order a divisor of $p^{\alpha}-1$ for some $\alpha \in \mathbb{N}_{0}$. A generator for this group will be denoted by $s_{H}$. Note that $s_{H} \in s_{\Delta}(G)$ (see, for example, $[\mathbf{1 5}, \S 8.2])$.

After this review of $[\mathbf{1 1}]$ we can establish the facts that we need to solve Problem 1.3. We define $L_{G} \in \mathbb{F}[V]$ by

$$
L_{G}=\prod_{H \in \mathcal{H} \Delta(G)} \ell_{s_{H}}^{\left|s_{H}\right|-1} .
$$

Note that $\operatorname{deg}\left(L_{G}\right) \leqslant\left|s_{\Delta(G)}\right|$, with equality if and only if $G$ contains no transvections.

Lemma 4.1. Let $\rho: G \hookrightarrow \operatorname{GL}(n, \mathbb{F})$ be a representation of a finite group $G$ over the field $\mathbb{F}$. Then $L_{G}$ divides any $\operatorname{det}^{-1}$-relative invariant.

Proof. The type of argument given by Stanley in [19, Theorem 3.1] is valid more generally. Specifically, let $f \in \mathbb{F}[V]_{\mathrm{det}^{-1}}^{G}$. Since the linear forms $\ell_{H^{\prime}}, \ell_{H^{\prime \prime}}$ are relatively prime for $H^{\prime} \neq H^{\prime \prime} \in \mathcal{H}_{\Delta}(G)$, it is enough to show that $\ell_{H}^{\left|s_{H}\right|-1}$ divides $f$ for each $H$ in $\mathcal{H}_{\Delta}(G)$. So let $H \in \mathcal{H}_{\Delta}(G)$ and choose an eigen basis $\ell_{H}=z_{1}, z_{2}, \ldots, z_{n}$ for $V^{*}$ so that the matrix of $s_{H}$ is of the form

$$
s_{H}=\left[\begin{array}{ccccc}
\lambda_{H} & & & & \\
0 & 1 & & & \\
& 0 & \ddots & 0 & \\
& & & & 1
\end{array}\right] \in \operatorname{GL}(n, \mathbb{F}) .
$$

Write $f$ as a sum of monomials in this basis, namely

$$
f=\sum_{i_{1} \neq 0} a_{I} z^{I}+\sum_{j_{1}=0} b_{J} z^{J}
$$

where $I=\left(i_{1}, \ldots, i_{n}\right)$ and $J=\left(j_{1}, \ldots, j_{n}\right)$ are multi-indices. Then

$$
s_{H} f=\sum_{i_{1} \neq 0} a_{I} \lambda_{H}^{i_{1}} z^{I}+\sum_{j_{1}=0} b_{J} z^{J}
$$

Since $s_{H} f=\lambda_{H}^{-1} f$, equating coefficients gives

$$
\begin{gathered}
i_{1} \geqslant\left|s_{H}\right|-1 \quad \text { if } a_{I} \neq 0, \\
b_{J}=0 \quad \text { for all } J
\end{gathered}
$$

and the result follows. 
Lemma 4.2. Let $\rho: G \hookrightarrow \operatorname{GL}(n, \mathbb{F})$ be a representation of a finite group $G$ over the field $\mathbb{F}$. Suppose that $\mathbb{F}[V]^{G}=\mathbb{F}\left[f_{1}, \ldots, f_{n}\right]$ is a polynomial algebra and $z_{1}, \ldots, z_{n} \in V^{*}$, then $L_{G}$ divides $J_{G}=\operatorname{det}\left[\partial f_{i} / \partial z_{j}\right]$.

Proof. Since the Jacobian is a $\operatorname{det}^{-1}$ invariant [15, p. 21], this follows from Lemma 4.1.

Lemma 4.3. Let $s \in \mathrm{GL}(n, \mathbb{F})$ be a pseudoreflection and let $\mathcal{H}$ be a finite set of hyperplanes in $V$. Suppose that

(i) $H_{s} \notin \mathcal{H}$, and

(ii) if $H \in \mathcal{H}$, then $s H \in \mathcal{H}$.

Let $a_{H} \in \mathbb{N}$ for $H \in \mathcal{H}$ with $a_{H}$ constant on each s-orbit and set $L=\prod_{H \in \mathcal{H}} \ell_{H}^{a_{H}}$. Then $s(L)=L$ and $\Delta_{s}(L)=0$.

Proof. Since $s$ permutes the hyperplanes in $\mathcal{H}$, it also permutes the collection of linear forms $\left\{\ell_{H} \mid H \in \mathcal{H}\right\}$ up to some non-zero scalar multiples, i.e. for $H^{\prime} \in \mathcal{H}$ there exists $H^{\prime \prime} \in \mathcal{H}$, and $\alpha\left(H^{\prime}\right) \in \mathbb{F}^{\times}$, such that $s\left(\ell_{H^{\prime}}\right)=\alpha\left(H^{\prime}\right) \ell_{H^{\prime \prime}}$. Therefore $s(L)=\alpha L$, where $\alpha=\prod_{H \in \mathcal{H}} \alpha(H)^{a_{H}} \in \mathbb{F}^{\times}$, and hence

$$
\ell_{s} \Delta_{s}(L)=(s-1)(L)=(\alpha-1) L .
$$

Since $H_{s} \notin \mathcal{H}$, the linear form $\ell_{s}$ is prime to $\ell_{H}$ for each $H \in \mathcal{H}$, and hence also to $L$. If $\alpha-1$ were not 0 , then this equation would imply, to the contrary, that $\ell_{s}$ divides $L$. Hence $\alpha-1=0$, and, since $\ell_{s} \neq 0$, we conclude that $\Delta_{s}(L)=0$.

Lemma 4.4. Let $\rho: G \hookrightarrow \operatorname{GL}(n, \mathbb{F})$ be a representation of a finite group over the field $\mathbb{F}$. Suppose that $\mathbb{F}[V]^{G}=\mathbb{F}\left[f_{1}, \ldots, f_{n}\right]$ is a polynomial algebra and

$$
L_{G}=\prod_{H \in \mathcal{H} \Delta(G)} \ell_{s_{H}}^{\left|s_{H}\right|-1}
$$

Then $L_{G}$ is a $\operatorname{det}^{-1}$-relative invariant.

Proof. Since $\mathbb{F}[V]^{G}$ is a polynomial algebra $G$ is generated by pseudoreflections (see [4, Chapter V, $\S 6$, Exercise 8]). We use the equivalent formula

$$
L_{G}=\prod_{H \in \mathcal{H} \Delta(G)} \ell_{s_{H}}^{\left|s_{H}\right|-1}
$$

For $H \in \mathcal{H}_{\Delta}(G)$ we find, after setting $L=L_{G} / \ell_{s_{H}}^{\left|s_{H}\right|-1}$,

$$
\begin{aligned}
s_{H}\left(L_{G}\right) & =s_{H}\left(\ell_{s_{H}}^{\left|s_{H}\right|-1}\right) s_{H}(L)=\lambda_{s_{H}}^{\left|s_{H}\right|-1} \ell_{s_{H}}^{\left|s_{H}\right|-1} L \quad(\text { by Lemma 4.3) } \\
& =\operatorname{det}\left(s_{H}\right)^{-1} \ell_{s_{H}}^{\left|s_{H}\right|-1} L=\operatorname{det}\left(s_{H}\right)^{-1} L_{G} .
\end{aligned}
$$


On the other hand, if $t \in s_{\Delta}(G)$ is a transvection, then setting $H=V^{t}$, recalling that $L_{G}=\ell_{s_{H}}^{\left|s_{H}\right|-1} L$, we have, from Lemma 4.3 ,

$$
t\left(L_{G}\right)=t\left(\ell_{s_{H}}^{\left|s_{H}\right|-1}\right) t(L)=\ell_{s_{H}}^{\left|s_{H}\right|-1} L
$$

since $t \ell_{s_{H}}=\ell_{s_{H}}$, as $\lambda_{t}=1=\operatorname{det}(t)$. The result follows because $s(G)$ generates $G$.

We require Hartmann's generalization of the formula of Benson and Crawley-Bovey $[\mathbf{3}, \mathbf{1 0}]$; her formulation runs as follows (see [7, Corollary 5]).

Proposition 4.5 (see $[7])$. Let $\rho: G \hookrightarrow \operatorname{GL}(n, \mathbb{F})$ be a representation of a finite group $G$ over the field $\mathbb{F}$ such that $\mathbb{F}[V]^{G}=\mathbb{F}\left[f_{1}, \ldots, f_{n}\right]$. Then

$$
\sum_{i=1}^{n}\left(\operatorname{deg}\left(f_{i}\right)-1\right) \geqslant \operatorname{deg}\left(L_{G}\right)
$$

with equality if and only if $G$ contains no transvections.

Theorem 4.6. Let $\rho: G \hookrightarrow \operatorname{GL}(n, \mathbb{F})$ be a representation of a finite group over the field $\mathbb{F}$ of characteristic $p$, which may be 0 . Suppose that $\mathbb{F}[V]^{G}=\mathbb{F}\left[f_{1}, \ldots, f_{n}\right]$ is a polynomial algebra. Then $J_{G}=\operatorname{det}\left[\partial f_{i} / \partial z_{j}\right]$ represents a fundamental class of $\mathbb{F}[V]_{G}$ if and only if $G$ contains no transvections.

Proof. Suppose that $G$ contains no transvections. Then, by [4, Chapter V, $\S 6$, Exercise 8], $s(G)=s_{\Delta}(G)$ generates $G$, so $G$ is generated by pseudoreflections of order relatively prime to the characteristic of $\mathbb{F}$. Therefore, by [11], the ideal of stable invariants $\mathcal{J}_{\infty}(G)$, and the ideal of generalized invariants associated to the set $s_{\Delta}(G), \mathcal{I}\left(s_{\Delta}(G)\right)$, coincide.

Let $\operatorname{deg}\left(f_{i}\right)=d_{i}$, for $i=1, \ldots, n$, so $J_{G}$ and a fundamental class for $\mathbb{F}[V]_{G}$ have the same degree, namely $\sum\left(d_{i}-1\right)$. This is also the degree of $L_{G}$ by Hartmann's result 4.5. Since $L_{G}$ divides $J_{G}$ by Lemma 4.2 , and $J_{G}$ is non-zero, it follows that they are nonzero multiples of each other. So it is sufficient to show that $L_{G} \neq 0$ in the algebra of coinvariants $\mathbb{F}[V]_{G}$. From $[\mathbf{1 1}]$ we have

$$
\left(\mathbb{F}[\bar{V}]^{G}\right)^{e}=\left(f_{1}, \ldots, f_{n}\right)=\mathcal{J}_{1}(G) \subseteq \cdots \subseteq \mathcal{J}_{\infty}(G)=\mathcal{I}\left(s_{\Delta}(G)\right),
$$

where, $\left(\mathbb{F}[\bar{V}]^{G}\right)^{e}$ denotes the extension to $\mathbb{F}[V]$ of the augmentation ideal of $\mathbb{F}[\bar{V}]^{G}$ of $\mathbb{F}[V]^{G}$, and the ideals $\mathcal{J}_{m}(G)$ are defined inductively (see $[\mathbf{1 1}, \S 1]$ ) by

$$
\mathcal{J}_{m}(G)= \begin{cases}(0) & \text { for } m=0 \\ \left(\left\{f \in \mathbb{F}[V] \mid g f-f \in J_{m-1}\right\}\right) & \text { for } m>0\end{cases}
$$

So it is more than enough to show that $L_{G} \notin \mathcal{I}\left(s_{\Delta}(G)\right)$. For this we evaluate

$$
\prod_{H \in \mathcal{H}_{\Delta}(G)} \Delta_{s_{H}}^{\left|s_{H}\right|-1}
$$


on $L_{G}$. We find, with $L$ as in the proof of Lemma 4.3, that, for $i \geqslant 1$,

$$
\Delta_{s_{H}}^{i}\left(L_{G}\right)=\Delta_{s_{H}}^{i}\left(\ell_{H}^{\left|s_{H}\right|-1} L\right)=\Delta_{s_{H}}^{i}\left(\ell_{s|H|-1}\right) L
$$

by Lemma 4.2 and the fact that $\Delta_{s_{H}}$ is a twisted derivation. Thus, by equation (4.2),

$$
\Delta_{s_{H}}^{\left|s_{H}\right|-1}\left(L_{G}\right)=\left(\lambda_{s_{H}}^{-1}-1\right) L
$$

Hence

$$
\left(\prod_{H \in \mathcal{H}_{\Delta}(G)} \Delta_{s_{H}}^{\left|s_{H}\right|-1}\right)\left(L_{G}\right)=\prod_{H \in \mathcal{H}_{\Delta}(G)}\left(\lambda_{s_{H}}^{-1}-1\right) !,
$$

and this scalar is non-zero. Therefore $L_{G} \notin \mathcal{I}\left(s_{\Delta}(G)\right)$, as required.

Conversely, if $s_{\Delta}(G) \neq \emptyset$ then the formula of Hartmann [7] gives

$$
\operatorname{deg}\left(J_{G}\right)>\sum\left(d_{i}-1\right)=\operatorname{deg}\left(L_{G}\right) .
$$

By Lemma $4.2, J_{G}=L_{G} \cdot K$ and $\operatorname{deg}(K)=\operatorname{deg}\left(J_{G}\right)-\operatorname{deg}\left(L_{G}\right)>0$. Since $L_{G}$ is a $\operatorname{det}^{-1}$-relative invariant by Lemma 4.4 , and so is $J_{G}$, it follows that $K$ must be invariant. Then $J_{G}=L_{G} \cdot K$ says that $J_{G} \in\left(f_{1}, \ldots, f_{n}\right)$, so $J_{G}$ is zero in $\mathbb{F}[V]_{G}$ and cannot represent a fundamental class.

Remark 4.7. Note that the proof shows that when $G$ contains no transvections then $\operatorname{det}\left[\partial f_{i} / \partial z_{j}\right]$ and $L_{G}$ are non-zero scalar multiples of each other, so $L_{G}$ also represents a fundamental class of $\mathbb{F}[V]_{G}$ if and only if $G$ contains no transvections.

Recall [9] that the transfer ideal of $\rho: G \hookrightarrow \operatorname{GL}(n, \mathbb{F})$ is the affine variety $\mathfrak{X}_{G} \subset V$ defined by the ideal $\operatorname{Im}\left(\operatorname{Tr}^{G}\right)^{e} \subset \mathbb{F}[V]$. It is the union of the fixed subspaces of the elements of order $p$ in $G$, and has dimension at most $n-1$. The only way it can have dimension $n-1$ is if $G$ contains transvections. Therefore we have the following corollary.

Corollary 4.8. Let $\rho: G \hookrightarrow \operatorname{GL}(n, \mathbb{F})$ be a representation of a finite group over the field $\mathbb{F}$ of characteristic $p$, which may be 0 . Suppose that $\mathbb{F}[V]^{G}=\mathbb{F}\left[f_{1}, \ldots, f_{n}\right]$ is a polynomial algebra. Then $\operatorname{det}\left[\partial f_{i} / \partial z_{j}\right]$ represents a fundamental class of $\mathbb{F}[V]_{G}$ if and only if the dimension of the transfer variety $\mathfrak{X}_{G}$ is at most $n-2$.

Acknowledgements. I am indebted to Julia Hartmann and Kathrin Kuhnigk for focusing my attention on these problems and tolerating my half-baked solutions: the extension of the formula of Benson and Crawley-Bovey [10] used here is also due to Julia Hartmann [7]. I thank John Hubbuck for his kind invitation to visit Aberdeen University, where I found the pleasant environment to bake things to completion. Thanks also go to the referee for useful criticism and simplifying the proof of Theorem 4.6 considerably. This work was done as part of a project financed by the British Engineering and Physical Sciences Research Council (EPSRC). 


\section{References}

1. J. F. Adams and C. W. Wilkerson, Finite $H$-spaces and algebras over the Steenrod algebra, Ann. Math. 111 (1980), 95-143.

2. D. J. Benson, Polynomial invariants of finite groups (Cambridge University Press, 1993).

3. D. J. Benson and W. W. Crawley-Boevey, A ramification formula for Poincaré series, and a hyperplane formula for modular invariants, Bull. Lond. Math. Soc. 27 (1995), 435440.

4. N. Bourbaki, Groupes et algèbres de Lie, Chapters 4, 5 and 6 (Masson, Paris, 1981).

5. C. Chevalley, Invariants of finite groups generated by reflections, Am. J. Math. 67 (1955), 778-782.

6. O. E. Glenn, Modular invariant processes, Bull. Am. Math. Soc. 21 (1914-1915), 167173.

7. J. HARTMAnN, Transvection free groups and invariants of polynomial tensor exterior algebras, Trans. Groups, in press.

8. G. Kemper, Calculating invariant rings of finite groups over arbitrary fields, J. Symbolic Comput. 21 (1996), 351-356.

9. K. Kuhnigk And L. Smith, Feshbach's transfer theorem and applications, preprint, AGInvariant Theory (1998).

10. A. Neeman, The connection between a conjecture of Carlisle and Kropholler, now a theorem of Benson and Crawley-Bovey, and Grothendieck's Riemann-Roch and duality theorems, Comment. Math. Helv. 70 (1995), 339-349.

11. F. Neumann, M. D. Neusel and L. Smith, Rings of generalized and stable invariants of finite groups, J. Algebra 182 (1996), 85-122.

12. M. D. NEusel, Inverse invariant theory and Steenrod operations, Memoirs of the American Mathematical Society, vol. 146, no. 692 (2000).

13. L. Sмiтh, Linear algebra (Springer, 1978, 2nd edn 1984, reprinted 1990, 1992, 3rd edn 1998).

14. L. Smith, A note on graded complete intersections, Q. J. Math. (2) 33 (1982), 379-384.

15. L. Sмiтh, Polynomial invariants of finite groups (Peters, Wellesley, MA, 1995, 2nd printing 1997).

16. L. Smith, $\mathcal{P}^{*}$-invariant ideals in rings of invariants, Forum Math. 8 (1996), 319-342.

17. L. Smith And R. M. Switzer, Realizability and nonrealizability of Dickson algebras as cohomology rings, Proc. Am. Math. Soc. 89 (1983), 303-313.

18. L. Smith And R. M. Switzer, Polynomial algebras over the Steenrod algebra, Proc. Edinb. Math. Soc. 27 (1984), 11-19.

19. R. P. Stanley, Relative invariants of finite groups generated by pseudoreflections, $J$. Algebra 49 (1977), 134-148.

20. C. W. WilKERSON, Inseparable forms of unstable polynomial algebras over the Steenrod algebra, preprint (available from ftp@hopf.math.purdue.edu). 
https://doi.org/10.1017/S0013091500000031 Published online by Cambridge University Press 